\title{
Spiral computed tomography for stratification of pulmonary embolism: ready for prime time?
}

\author{
Guy Meyer and Olivier Sanchez
}

Affiliations: Division of Respiratory and Intensive Care, Hôpital Européen Georges Pompidou, AP-HP; Université Paris Descartes, Sorbonne Paris Cité, INSERM UMR S 970, Paris, France.

Correspondence: G. Meyer, Service de Pneumologie, Hôpital Européen Georges Pompidou, 20 rue Leblanc, 75015 Paris, France. E-mail: guy.meyer@egp.aphp.fr

0

@ERSpublications

Should spiral CT be used for risk stratification of all patients with pulmonary embolism?

http://ow.ly/ulS3k

The risk stratification of patients with pulmonary embolism has been the subject of a large number of studies in recent years. Current guidelines suggest that patients be stratified in order to identify low-risk patients, who might benefit from outpatient treatment, and high-risk patients, who should be admitted to hospital and who might benefit from more aggressive forms of treatment [1]. This can be achieved by clinical examination alone using either an explicit risk stratification tool or a more empirical set of criteria [2-5]. Subclinical myocardial injury and right ventricular dysfunction, assessed by biomarkers, echocardiography or spiral computed tomography, also have been shown to be associated with an increased risk of an adverse outcome in patients with pulmonary embolism [6-8].

The study by BECATTINI et al. [9], published in this issue of the European Respiratory Journal, reinforces the role of computed tomography (CT) in the risk stratification of patients with pulmonary embolism. Using a systematic review and meta-analysis of all retrospective and prospective studies assessing the role of $\mathrm{CT}$ for the prognosis of patients with acute pulmonary embolism, BECATTINI et al. [9] were able to demonstrate or to confirm that right ventricular dysfunction is associated with an increased risk of adverse outcome in patients with pulmonary embolism. Indeed, right ventricular dysfunction was associated with overall death at 1 and 3 months in all patients with pulmonary embolism and in clinically stable patients. Right ventricular dysfunction was also associated with the outcome of death due to pulmonary embolism. These results were observed for reconstructed four-chamber images and for transverse bi-dimensional images, suggesting that the former technique is not mandatory for the assessment of right ventricular dysfunction on spiral CT.

Previous work by the same group demonstrated high interobserver agreement (kappa-statistic 0.88 ) and diagnostic accuracy (area under the curve 0.86 ) of spiral CT for detecting right ventricular dysfunction, by using echocardiography as the reference standard [10]. Although echocardiography can provide the same information on right ventricular dysfunction, it is not available in noncardiology departments, whereas spiral CT is more widely available and can provide clinicians with both diagnostic and prognostic information for patients with pulmonary embolism.

The information provided by systematic reviews and meta-analyses is critically dependent on the limitations of the original studies included in the analysis. In most of the studies included in the meta-analysis reported by BECATTINi et al. [9], the results of spiral CT were not adjusted for clinical variables and biomarkers. Accordingly, these results do not demonstrate that spiral CT provides independent prognostic information

Received: Feb 262014 | Accepted after revision: March 032014

Conflict of interest: Disclosures can be found alongside the online version of this article at www.erj.ersjournals.com

Copyright @ERS 2014 
to clinical findings and biomarkers. Previous studies suggest that right ventricular dysfunction diagnosed on echocardiography adds significant prognostic information to clinical findings and the pulmonary embolism severity index (PESI) [11, 12], but this has not been reported in all studies [13]. Clinical risk stratification tools reflect both the clinical severity of pulmonary embolism and the underlying disease, and probably provide different information than spiral CT and biomarkers.

What can we conclude from these findings? From an epidemiological point of view, right ventricular dysfunction is undoubtedly associated with an increased risk of mortality in patients with pulmonary embolism, but the question remains if spiral CT adds significant prognostic information to biomarkers and clinical findings.

From a physiological point of view, the results reported by BECATTINI et al. [9] reinforce some rather old but pioneering data suggesting that the prognosis of pulmonary embolism is not related to the level of pulmonary vascular obstruction per se, but to the consequences on the right ventricular function [14]. Indeed, in a similar analysis conducted by the same group, pulmonary vascular obstruction was not associated with mortality in patients with pulmonary embolism [15].

From a practical point of view, the evaluation of right ventricular dilation on transverse images provided by spiral CT is easy to perform. Right ventricular dilation is associated with an increased risk of adverse outcome and should prompt the measurement of cardiac biomarkers. Patients with both right ventricular dilation and increased blood levels of cardiac biomarkers belong to the intermediate-risk group as defined by the European Society of Cardiology [1]. Our own recent, unpublished data suggest that thrombolytic treatment may decrease the rate of haemodynamic decompensation in these patients and may be considered when the bleeding risk is low. Conversely, the absence of right ventricular dilation on spiral CT is associated with a low rate of adverse outcome related to pulmonary embolism and these patients can benefit from early discharge, provided that no underlying condition requires hospitalisation. The question remains if right ventricular dysfunction should be measured in all patients with confirmed pulmonary embolism, or only in patients belonging to PESI class III or IV or with sPESI $\geqslant 1$.

\section{References}

1 Torbicki A, Perrier A, Konstantinides S, et al. Guidelines on the diagnosis and management of acute pulmonary embolism: the Task Force for the Diagnosis and Management of Acute Pulmonary Embolism of the European Society of Cardiology (ESC). Eur Heart J 2008; 29: 2276-2315.

2 Aujesky D, Obrosky DS, Stone RA, et al. Derivation and validation of a prognostic model for pulmonary embolism. Am J Respir Crit Care Med 2005; 172: 1041-1046.

3 Jimenez D, Aujesky D, Moores L, et al. Simplification of the pulmonary embolism severity index for prognostication in patients with acute symptomatic pulmonary embolism. Arch Intern Med 2010; 170: 1383-1389.

4 Zondag W, Mos IC, Creemers-Schild D, et al. Outpatient treatment in patients with acute pulmonary embolism: the Hestia Study. J Thromb Haemost 2010; 9: 1500-1507.

5 Erkens PM, Gandara E, Wells P, et al. Safety of outpatient treatment in acute pulmonary embolism. $J$ Thromb Haemost 2010; 8: 2412-2417.

6 Sanchez O, Trinquart L, Colombet I, et al. Prognostic value of right ventricular dysfunction in patients with haemodynamically stable pulmonary embolism: a systematic review. Eur Heart J 2008; 29: 1569-1577.

7 Klok FA, Mos IC, Huisman MV. Brain-type natriuretic peptide levels in the prediction of adverse outcome in patients with pulmonary embolism: a systematic review and meta-analysis. Am J Respir Crit Care Med 2008; 178: 425-430.

8 Becattini C, Vedovati MC, Agnelli G. Prognostic value of troponins in acute pulmonary embolism: a meta-analysis. Circulation 2007; 116: 427-433.

9 Becattini C, Agnelli G, Germini F, et al. Computed tomography to assess risk of death in acute pulmonary embolism: a meta-analysis. Eur Respir J 2014; 43: 1678-1690.

10 Becattini C, Agnelli G, Vedovati MC, et al. Multidetector computed tomography for acute pulmonary embolism: diagnosis and risk stratification in a single test. Eur Heart J 2011; 32: 1657-1663.

11 Sanchez O, Trinquart L, Caille V, et al. Prognostic factors for pulmonary embolism: the prep study, a prospective multicenter cohort study. Am J Respir Crit Care Med 2010; 181: 168-173.

12 Sanchez O, Trinquart L, Planquette B, et al. Echocardiography and pulmonary embolism severity index have independent prognostic roles in pulmonary embolism. Eur Respir J 2013; 42: 681-688.

13 Lankeit M, Gomez V, Wagner C, et al. A strategy combining imaging and laboratory biomarkers in comparison to a simplified clinical score for risk stratification of patients with acute pulmonary embolism. Chest 2012; 141: 916-922.

14 Alpert JS, Smith R, Carlson J, et al. Mortality in patients treated for pulmonary embolism. JAMA 1976; 236: $1477-1480$.

15 Vedovati MC, Becattini C, Agnelli G, et al. Multidetector CT scan for acute pulmonary embolism: embolic burden and clinical outcome. Chest 2013; 142: 1417-1424. 